\title{
Material Classification for Printed Circuit Boards by Kernel Fisher Discriminant Analysis
}

\author{
Takahiko Horiuchi, Yuhei Suzuki, and Shoji Tominaga \\ Graduate School of Advanced Integration Science, Chiba University, Japan \\ \{horiuchi@faculty, suzukiyuhei@graduate, shoji@faculty\}.chiba-u.jp
}

\begin{abstract}
This paper proposes an approach to a reliable material classification for printed circuit boards by kernel Fisher discriminant analysis. The proposed approach uses only three dimensional features of the surface-spectral reflectance reduced from the high-dimensional spectral imaging data for effectively classifying the surface material on each pixel point into several elements such as substrate, metal, resist, footprint, and silk-screen paint. We show that a linear classification of these elements does not work well, because the feature distribution is not well separated in the three dimensional feature space. In this paper, a kernel technique is used to constructs a subspace where the class separability is maximized in a high-dimensional feature space. The performance of the proposed method is compared with the previous algorithms using the high-dimensional spectral data.
\end{abstract}

Keywords: Material classification, printed circuit board, spectral reflectance, region segmentation, kernel discriminant analysis.

\section{Introduction}

Automatic visual inspection (AVI) has become crucial to improve quality in printed circuit board (PCB) manufacture. A PCB is one of the most complicated minute objects to understand from the observed image in a variety of industries. The raw $\mathrm{PCB}$, i.e. boards without components, may have defects such as: hairline, pin-hole, wrong size hole, open circuit, and breakout. Many researchers (e.g., see the references in [1]) repeatedly emphasized the importance of developing techniques and algorithms for an automatic inspection system in the electronic industry. Consequently, a wide range of defect detection techniques and algorithms have been reported and implemented in AVI systems [2-6]. Most of them were based on binary or gray-scale images to find board defects. A raw PCB surface layer is composed of various elements, which are a mixture of different materials, and the area of each element is very small. These features make the machine inspection difficult by using binary, gray-scale images, or even by using general color imaging systems based on only three spectral bands of RGB. The segmentation of a PCB image enables us to transform the original problem of inspecting a complex PCB image to a simpler problem of inspecting a well-defined segmented image. The surface of the raw PCB is partitioned into small areas of different materials such as metal, resist, footprint, 
substrate, and print areas. These materials are difficult to be classified accurately based on binary, gray-scale or RGB images.

In order to solve the problems, in our previous works, a material classification algorithm was proposed based on surface-spectral reflectance [7-10]. Those techniques can produce higher classification accuracy comparing with grayscalebased or RGB-based approaches. However, the analysis faces the problem of data storage requirement and high-computation cost, because of high-dimension of spectral data. Dimensional reduction of spectral data is important for improving the high-computation cost.

The present paper proposes an approach to a reliable material classification for PCBs by kernel Fisher discriminant analysis. The proposed approach uses only 3channel signals of the surface spectral reflectance selected from the high-dimensional spectral imaging data for effectively classifying the surface material on each pixel point into several elements. The performance of our spectral image segmentation algorithm is compared with the previous segmentation algorithms using highdimensional spectral imaging data. Experimental results from a number of raw PCBs have shown the effectiveness of the developed method for classification of complicated images.

\section{Imaging System}

\subsection{Spectral Camera System}

Figure 1 shows the constructed spectral imaging system for capturing raw PCBs. The camera system consists of a monochromatic CCD camera (Retiga 1300) with 12-bit dynamic range and Peltier cooling, a macro lens of C-mount connected directly to the camera, VariSpec ${ }^{\mathrm{TM}}$ Liquid Crystal Tunable Filter (LCTF), IR-cut filter and a personal computer. The LCTF is convenient for spectral imaging because the wavelength band can be changed easily and electronically. The LCTF used in this study has the spectral properties of narrow band filtration of $10 \mathrm{~nm}$ and wavelength range [400-700 nm]. The XY stage helps to easily control the camera system distance and position. The rotating stage controls rotation and position of the measured object. The imaging system automatically captures and saves spectral images with arbitrary number of bands and shutter speeds. The actual measurement time required for capturing one spectral image with size $1280 \times 1024$ pixels for the area $35 \mathrm{~mm} \times 30 \mathrm{~mm}$ and 31-bands is 4.75 seconds. The viewing direction of the camera is always perpendicular to the board surface as shown in Fig. 1.

\subsection{Observation Condition}

Suitable lighting and viewing conditions can facilitate inspections, avoiding the needs for complex image processing algorithms. In this study, we use multiple light sources of $300 \mathrm{~W}$ incandescent lamps. Figure 2 shows the observation geometry with two light sources for effective surface illumination. In order to avoid large fluctuation of pixel 
values between highlight area and matte area, we control the illumination from two directions [left and right]. The light sources illuminate the same surface alternatively from one of the two directions that are mirrored about the viewing direction. Each light source is carefully set to illuminate the surface of the PCB uniformly. We investigated a proper illumination angle for observing PCB materials. We found that the minimum illumination angle is 20-degrees and this is because of the camera shadow on the board. Decreasing the incident angle to less than 25-degrees makes strong specular highlight on the board especially on metal parts, and increasing this angle to more than 25-degrees makes metal parts more noisy and difficult to classify. The illumination angles were determined empirically as 25-degrees to the surface normal of the board. The viewing direction of the camera was perpendicular to the board surface. The board is placed a few $\mathrm{cm}$ apart from the front of the camera system.

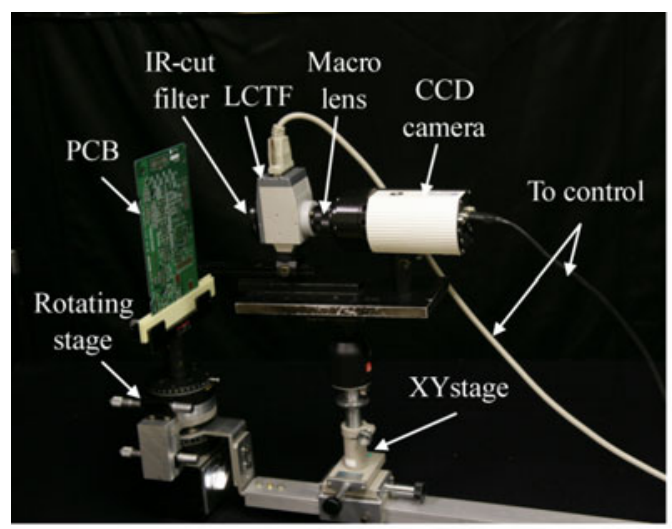

Fig. 1. Imaging system

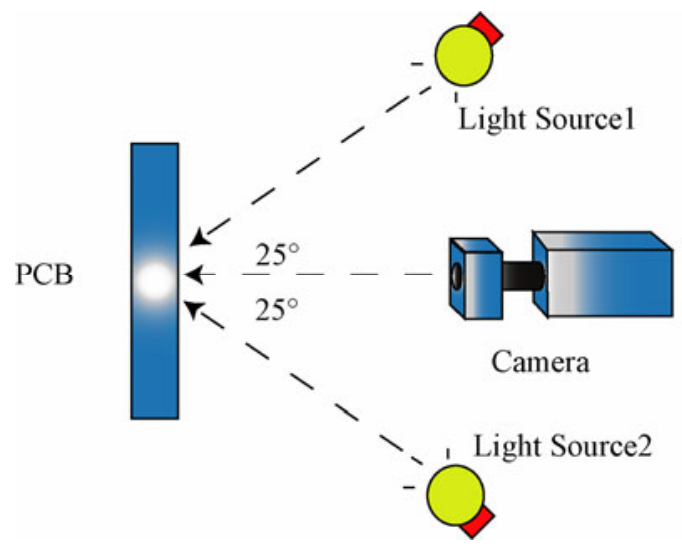

Fig. 2. Observation geometry with multiple light sources 


\subsection{Reflection Properties of Printed Circuit Board}

The observed surface reflection depends not only on the material composition, but also on the geometry of observation. For instance, the metal surface includes strong specular highlight, depending on viewing and lighting angles. Sharp edges of metal flakes and holes produce specular highlight effect on one side and shadowing effect on the other side. In addition, the footprint is a sort of metal composed of solder. This object surface is rough, and gloss appears on the surface at some angles of viewing and lighting. These properties lead to large fluctuation of pixel values between highlight area and matte area. Therefore our acquisition system with different illumination directions is useful for discriminating the surface appearances. Figure 3 illustrates a part of printed circuit board captured by our system and contains the elements of metal, substrate (base material of the board), print (silk-screen print), footprint (gray metallic footprint), and resist (metals coated with photo-resist).

While the camera system dynamic range of 12-bits is adequate for most parts of circuit board surfaces, it is often needed to acquire HDR images for some parts including specular highlights such as metal. Three pictures of the same scene are automatically taken with different shutter speeds (different exposures), and the multiple images are combined into a single image to extend the dynamic range of the camera to 14-bits. Thus we can capture the spectral data of minute printed circuit board objects from the HDR images at different illumination directions.

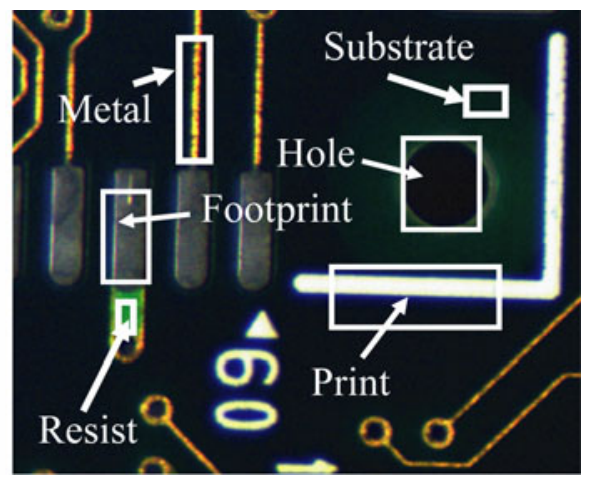

Fig. 3. Partial image of a raw circuit board

\subsection{Estimation of Surface-Spectral Reflectance}

We use a straightforward way for obtaining reliable estimates of the surface-spectral reflectance function from the camera outputs under narrow band filtration. Let $\lambda_{1}, \lambda_{2}, \ldots, \lambda_{\rho}$ be $\rho$ wavelengths of filtration, usually corresponding to 400 , $410, \ldots, 700 \mathrm{~nm}$, and let $S(\lambda)$ be the surface-spectral reflectance. In our measurement system, $\rho=31$ at $10 \mathrm{~nm}$ interval. The spectral reflectance can be estimated from the sensor outputs $O_{k}$ as 


$$
S\left(\lambda_{k}\right)=\frac{O_{k}}{\int_{400}^{700} E(\lambda) R_{k}(\lambda) d \lambda}, \quad(k=1,2, \ldots, \rho)
$$

where $E(\lambda)$ is the illuminant spectral-power distribution of an incandescent lamp, and $R_{k}(\lambda)$ is the $k$-th sensor spectral sensitivity function. The reflectance image without specular highlight and shadow are needed for material classification of raw circuit boards. That is, a light source illuminates the same surface alternatively from two directions [left and right]. We then unify the spectral reflectance data to produce only one spectral reflectance image from the captured images by the algorithm in Refs. [9-10].

\section{Classification Algorithm with Dimensional Reduction}

We consider dimension reduction for classification based on Fisher discriminant analysis.

\subsection{Fisher Discriminant Analysis}

The Fisher linear discriminant analysis (FLDA) is known as effective feature extraction and class discrimination analysis for 2-class or multi-class problem in pattern recognition and data analysis. In the following we first apply Fisher's discriminant to the PCB classification.

Let $\mathbf{s}_{i}=\left\{S\left(\lambda_{1}\right), S\left(\lambda_{2}\right), S\left(\lambda_{3}\right)\right\}_{i}$ be a three-dimensional surface reflectance vector which is properly sampled from $\rho$-dimensional estimated reflectance in Eq.(1). A collection of training samples $\left\{\mathbf{s}_{i}\right\}$ is denoted as $\chi=\left\{\mathbf{s}_{1}, \mathbf{s}_{2}, \cdots, \mathbf{s}_{n}\right\}$. Suppose that the sample $\mathbf{s}_{i}$ belong to the $l_{i}$-th class, $l_{i} \in\{1,2, \cdots, L\}$ and $n_{l}$ is the number of samples belonging to the $l$-th class. In this paper, $L=5$ (substrate, metal, resist, footprint, and paint).

Given a three-dimensional surface reflectance vector $\mathbf{s}$, the Fisher's linear discriminant is then obtained by a new feature vector $\mathbf{w}=\mathbf{A}^{T} \mathbf{S}$ which maximizes

$$
J(\mathbf{w})=\frac{\mathbf{w}^{T} \boldsymbol{\Sigma}_{B} \mathbf{w}}{\mathbf{w}^{T} \boldsymbol{\Sigma}_{W} \mathbf{w}}
$$

where $\mathbf{A}=\left[a_{i j}\right]$ is a coefficient matrix. The matrix $\boldsymbol{\Sigma}_{W}$ and $\boldsymbol{\Sigma}_{B}$ are the within-class covariance matrix and the between-class covariance matrix of the input feature vectors $\mathbf{s}$, which are computed as

$$
\begin{aligned}
& \boldsymbol{\Sigma}_{W}=\sum_{i=1}^{n} \alpha_{l_{i}}\left(\mathbf{s}_{i}-\overline{\mathbf{s}}_{l_{i}}\right)\left(\mathbf{s}_{i}-\overline{\mathbf{s}}_{l_{i}}\right)^{T}, \\
& \boldsymbol{\Sigma}_{B}=\sum_{l=1}^{L} \alpha_{l}\left(\overline{\mathbf{s}}_{l}-\overline{\mathbf{s}}_{T}\right)\left(\overline{\mathbf{s}}_{l}-\overline{\mathbf{s}}_{T}\right)^{T},
\end{aligned}
$$


where $\alpha_{l}, \overline{\mathbf{s}}_{l}$ and $\overline{\mathbf{s}}_{T}$ denote a priori probability of class- $l$, the mean vector of the mapped samples belonging to the class- $l$, and the mean vector of all mapped samples, respectively.

The optimal coefficient matrix $\mathbf{A}$ is then given by solving the following eigen-equation

$$
\boldsymbol{\Sigma}_{B} \mathbf{A}=\boldsymbol{\Sigma}_{W} \mathbf{A} \mathbf{\Lambda}, \quad\left(\mathbf{A}^{T} \boldsymbol{\Sigma}_{W} \mathbf{A}=\mathbf{I}\right),
$$

where $\boldsymbol{\Lambda}$ is a diagonal matrix of eigenvalues and $\mathbf{I}$ denotes the unit matrix. The $j$-th column of $\mathbf{A}$ is the eigenvector corresponding to the $j$-th largest eigenvalue. Thus, importance of each element of the new features $\mathbf{w}$ are evaluated by the corresponding eigenvalues.

We applied FLDA to the PCB classification. However, linear classification did not work well in this case. Figure 4 shows an example of feature distribution when $\lambda_{1}=450 \mathrm{~nm}, \lambda_{2}=550 \mathrm{~nm}$ and $\lambda_{3}=650 \mathrm{~nm}$. We can find specific characteristics for the average reflectance of each element. For example, $\lambda_{1}, \lambda_{2}$ and $\lambda_{3}$ are high reflectance for the print, and $\lambda_{2}$ and $\lambda_{3}$ are high reflectance but $\lambda_{1}$ is low reflectance for the metal. However, those three dimensional features are distributed and mixed by the low reflectance part. Therefore, clearly we cannot classify the overlapped clusters. If we transform the feature distribution into a higher dimensional space, linear classification can be possible. Linear classification in a higher dimensional feature space is developed with low-computational cost by using a kernel trick technique in the next section.

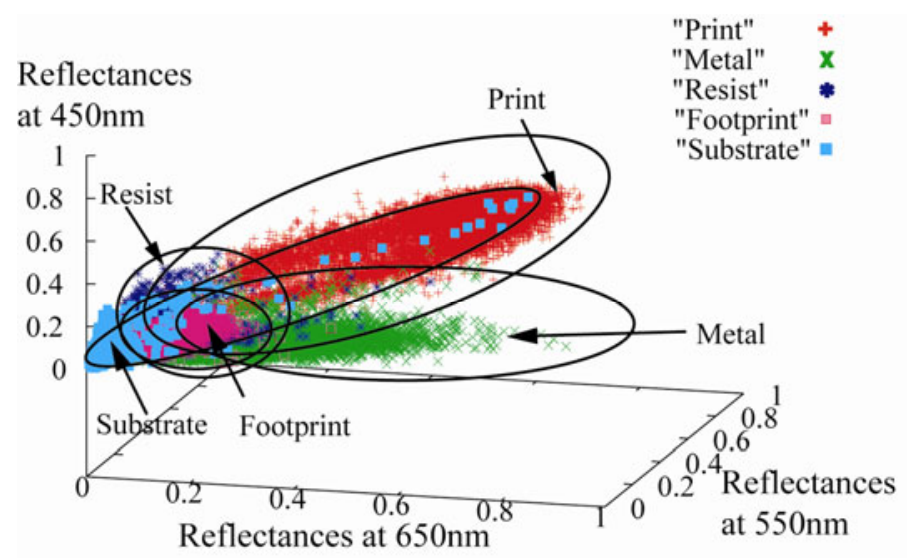

Fig. 4. Feature distribution in three dimensional feature space

\subsection{Classification by Kernel Fisher Discriminant Analysis}

Let $\phi: \mathbf{s} \in \chi \rightarrow \phi(\mathbf{s}) \in F$ be a nonlinear mapping from the original input space to a higher dimensional feature space $F$. 
The main idea of Kernel Fisher discriminant analysis (KFDA) is to apply the Fisher criterion in the higher dimensional feature space. So the criterion for KFDA algorithm can be given by the new feature vector $\psi$ which maximizes

$$
\begin{gathered}
J(\boldsymbol{\psi})=\frac{\boldsymbol{\psi}^{T} \boldsymbol{\Sigma}_{B}^{(K)} \boldsymbol{\psi}}{\boldsymbol{\psi}^{T} \boldsymbol{\Sigma}_{W}^{(K)} \boldsymbol{\psi}}, \\
\boldsymbol{\psi} \in \operatorname{span}\left\{\phi\left(\mathbf{s}_{i}\right), i=1, \cdots, n\right\} \subset F, \boldsymbol{\psi}=\sum_{i=1}^{n} a_{i} \phi\left(\mathbf{s}_{i}\right),
\end{gathered}
$$

where $a_{i}$ is the coefficient for the $i$-th kernel base. Clearly, if $F$ is very high- or even infinite dimensional, this will be impossible to solve directly. To overcome this limitation, dot-products $(\phi(\boldsymbol{\omega}) \cdot \phi(\mathbf{s}))$ of the training patterns are used instead of mapping the data explicitly. As we are then able to compute these dot-products efficiently, we can solve the original problem without ever mapping explicitly to $F$. This can be achieved Mercer kernels. These kernels $K(\boldsymbol{\omega}, \mathbf{s})$ compute a dot-product in some feature space $F$, i.e. $K(\boldsymbol{\omega} \cdot \mathbf{s})=(\phi(\boldsymbol{\omega}) \cdot \phi(\mathbf{s}))$. Then the Eq.(7) can be written as

$$
\boldsymbol{\psi}=\sum_{i=1}^{n} \mathbf{a}_{i} K\left(\boldsymbol{\omega}_{i}, \mathbf{s}\right),
$$

where $\mathbf{a}_{i}$ is the coefficient vector for the $i$-th kernel base.

Let $\mathbf{K}(\mathbf{s})=\left(K\left(\boldsymbol{\omega}_{1}, \mathbf{s}\right), \cdots, K\left(\boldsymbol{\omega}_{n}, \mathbf{s}\right)\right)^{T}$ be a vector of kernel bases for a feature vector $\mathbf{s}$. Then the Eq.(8) can be written as

$$
\boldsymbol{\psi}=\mathbf{A}^{(K)^{T}} K(\mathbf{s}),
$$

where $\mathbf{A}^{(K)^{T}}=\left[\mathbf{a}_{1}, \cdots, \mathbf{a}_{n}\right]$ is the coefficient matrix. As a kernel function, in this paper, an isotropic Gaussian function $K\left(\boldsymbol{\omega}_{i}, \mathbf{s}\right)=\exp \left[-\left\|\boldsymbol{\omega}_{i}-\mathbf{s}\right\|^{2} / 2 \sigma^{2}\right]$ is typically used. The location of the kernel base $\omega_{i}$ is fixed to each of the training samples and the number of kernel $n$ equals to the number of the training samples. Moreover the matrix $\boldsymbol{\Sigma}_{W}^{(K)}$ and $\boldsymbol{\Sigma}_{B}^{(K)}$ are the within-class covariance matrix and the between-class covariance matrix of the kernel vectors $\mathbf{K}(\mathbf{s})$, which are computed as

$$
\begin{aligned}
& \boldsymbol{\Sigma}_{W}^{(K)}=\sum_{i=1}^{n} \alpha_{l_{i}}\left(\mathbf{K}\left(\mathbf{s}_{i}\right)-\overline{\mathbf{K}\left(\mathbf{s}_{l_{i}}\right)}\right)\left(\mathbf{K}\left(\mathbf{s}_{i}\right)-\overline{\mathbf{K}\left(\mathbf{s}_{l_{i}}\right)}\right)^{T}, \\
& \boldsymbol{\Sigma}_{B}^{(K)}=\sum_{l=1}^{L} \alpha_{l}\left(\overline{\mathbf{K}\left(\mathbf{s}_{l}\right)}-\overline{\mathbf{K}\left(\mathbf{s}_{T}\right)}\right)\left(\overline{\mathbf{K}\left(\mathbf{s}_{l}\right)}-\overline{\mathbf{K}\left(\mathbf{s}_{T}\right)}\right)^{T},
\end{aligned}
$$

where $\overline{\mathbf{K}\left(\mathbf{s}_{l}\right)}$ and $\overline{\mathbf{K}\left(\mathbf{s}_{T}\right)}$ denote the mean vector of the mapped samples belonging to the class- $l$, and the mean vector of all mapped samples, respectively. 
The optimal coefficient matrix $\mathbf{A}^{(K)}$ is then given by solving the following eigen-equation

$$
\boldsymbol{\Sigma}_{B}^{(K)} \mathbf{A}^{(K)}=\boldsymbol{\Sigma}_{W}^{(K)} \mathbf{A} \mathbf{\Lambda}, \quad\left(\mathbf{A}^{(K) T} \boldsymbol{\Sigma}_{W}^{(K)} \mathbf{A}^{(K)}=\mathbf{I}\right)
$$

Figure 5 shows two examples of new feature vector $\boldsymbol{\psi}$ and projected features in Fig.4. To show in the figure easily, a binary relation is plotted. By the linear classification in the high-dimensional space, each element can be classified appropriately.

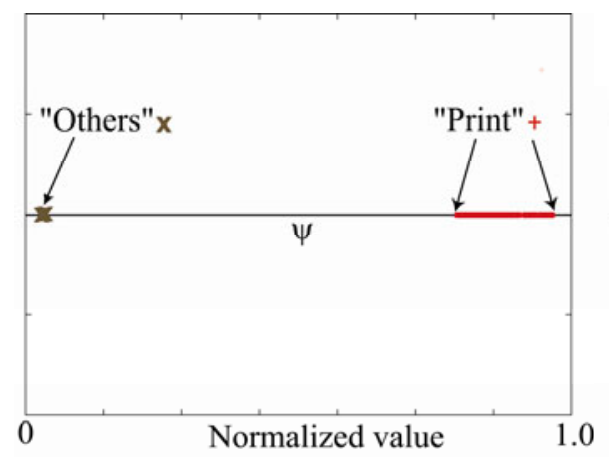

(a) Print v.s. Others

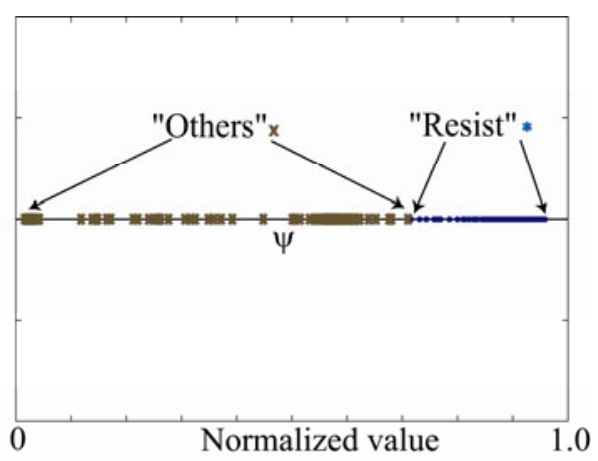

(b) Resist v.s. Others

Fig. 5. Projected features on the new feature vector $\psi$ in the high-dimensional space

\section{Experiments}

We have examined the performance of the proposed method in experiments using real samples of raw PCBs. In our experiments, we used three surface reflectance values at $\lambda_{1}=450 \mathrm{~nm}$ in blue region, $\lambda_{2}=550 \mathrm{~nm}$ in green region, and $\lambda_{3}=650 \mathrm{~nm}$ in red region as three-dimensional features. In our preliminary experiment, we examined various order of the classification such as simultaneous classification $(L=5)$ and serious binary classification $(L=2)$. As a result we selected the binary classification by classifying one by one. Concretely, we classified the PCB elements in order of metal, print, resist, footprint and substrate. As training samples $\chi$ for designing classifier, we selected 100 samples from the target element and 100 samples from other elements at random. Therefore, in the present experiment, we set $n=200$ and $L=2$ for each classification process. The standard deviations of Gaussian kernel were set $\sigma=0.2$ for print and metal, $\sigma=0.4$ for footprint and $\sigma=0.8$ for resist. The influence of these parameters to the classification result is discussed in Sec. 6.

The scene of a raw circuit board in Fig. 3 was captured with the present spectral imaging system under incandescent lamps. The image size was $1137 \times 867$ pixels. Two data sets of surface-spectral reflectances were estimated from the two spectral images at two different light sources. We combined these reflectance images into one reflectance image by comparing the corresponding reflectances at the same pixel point 
and applying the above rules to all pixels. Fig. 6(a) shows the typical spectral reflectance image obtained for the PCB in Fig. 3. Next, the proposed classification algorithm was executed for the spectral reflectance image. Figure 6(b) shows the classification results by using the three-dimensional data. In the figure, the segmented are painted in different colors, such as white for print, yellow for metal, green for resist-coated metal, black for substrate and grey for footprint. It should be note that the observed PCB image is clearly classified into five material regions and through-holes.

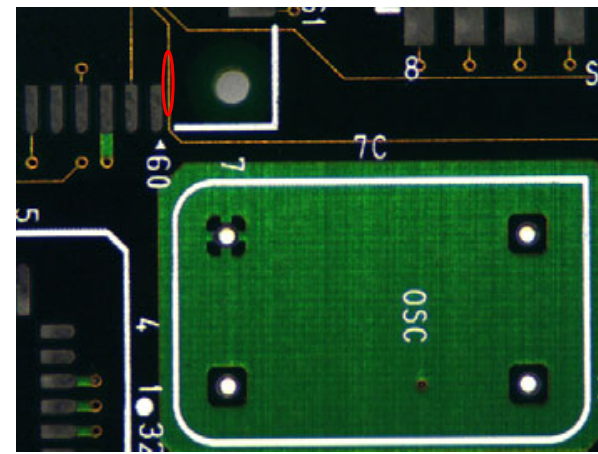

(a) Reflectance image

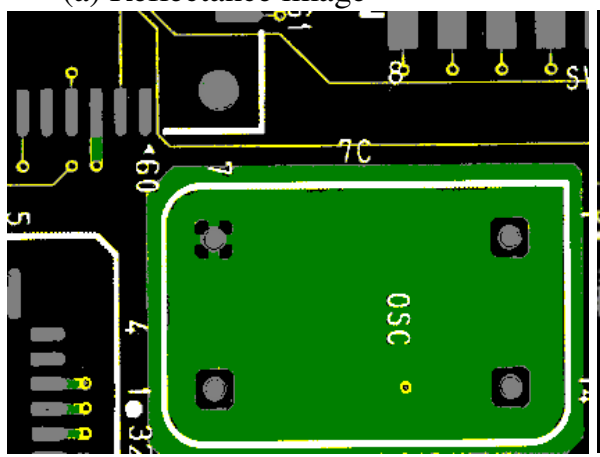

(c) Ref.[10] (31 dim.)

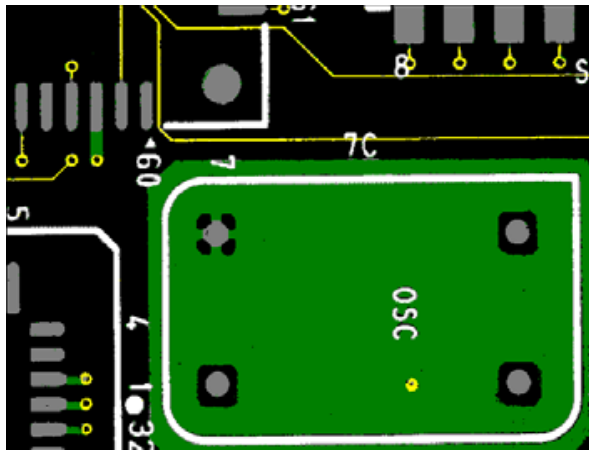

(b) Proposed classification (3 dim.)

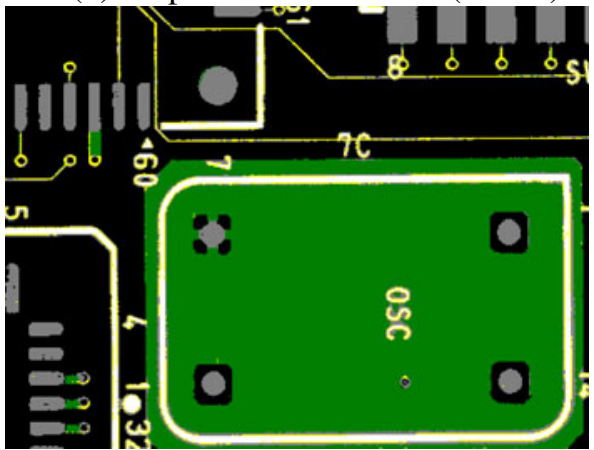

(d) k-means (31 dim.)

Fig. 6. Material classification results for a part of the raw PCB $(1137 \times 867)$

For comparison with the previous algorithms using high-dimensional spectral features, we choose the normalized cut [10] and the traditional k-means algorithm. Those algorithms do not need training samples, but they require expensive computational cost and memory requirements for large size images. The final segmentation results for all algorithms are summarized in Fig. 6. Figs. 6(c) and (d) show the segmentation results by the normalized cut algorithm and k-means clustering, respectively. We changed the initial seed points for k-means many times but we obtained almost the same result. Figures 7(a)-(d) show close-up images of Figs.6(a)-(d) at a thin metal line in the red-circle region in Fig. 6(a). It is obvious in the close-up view that the proposed method provides better classification even in thin lines than the other methods. Note that a lot of thin metal lines were classified correctly by the proposed method. 


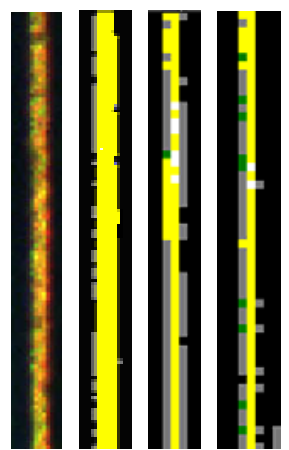

(a) (b) (c) (d)

Fig. 7. Close-up images of a thin metal line of Fig.6

The accuracy of material classification is demonstrated by comparing the classification results with the ground truth of each circuit board. Table 1 lists the accuracy of the algorithms used in comparison. The classification by the respective methods was repeated with different parameter settings. The table shows the best classification results for comparison. The classification quality for the whole regions is calculated numerically using

$$
\text { Quality rate }=\frac{\text { Correct classified pixels }}{\text { Total number of pixels }} \text {. }
$$

As in Table 1, the accuracy of the proposed supervised algorithm with threedimensional features has almost the same quality as our previous unsupervised algorithm with 31-dimensional features.

Table 1. Comparison of the accuracy for the compared methods

\begin{tabular}{|c|c|c|c|}
\hline Method & $\begin{array}{c}\text { Proposed } \\
\text { method }\end{array}$ & $\begin{array}{c}\text { Previous } \\
\text { method [10] }\end{array}$ & $\begin{array}{c}\text { 31dim. } \\
\text { k-means }\end{array}$ \\
\hline Quality rate & $96.4 \%$ & $96.3 \%$ & $93.5 \%$ \\
\hline
\end{tabular}

\section{Discussion}

The accuracy can depend on the number of training samples, the size of the PCBs, the standard deviation of kernel functions, and the feature selection. By changing these parameters, we have investigated the performance of the proposed algorithm.

\subsection{The Number of Samples}

Figure 8 shows the relationship between the number of training samples and the quality rate. The training samples were selected at random from the data set, and the same experiment was performed ten times in the number of each sample. The other experimental conditions were the same as Sec. 4. The result shows that high quality 
rate around $95 \%$ can be obtained even by ten samples if the samples are appropriately given. However, the quality rate is greatly influenced with the training sample. The results are unstable when using a small number of training samples. In other words, more than 50 samples are needed for high quality.

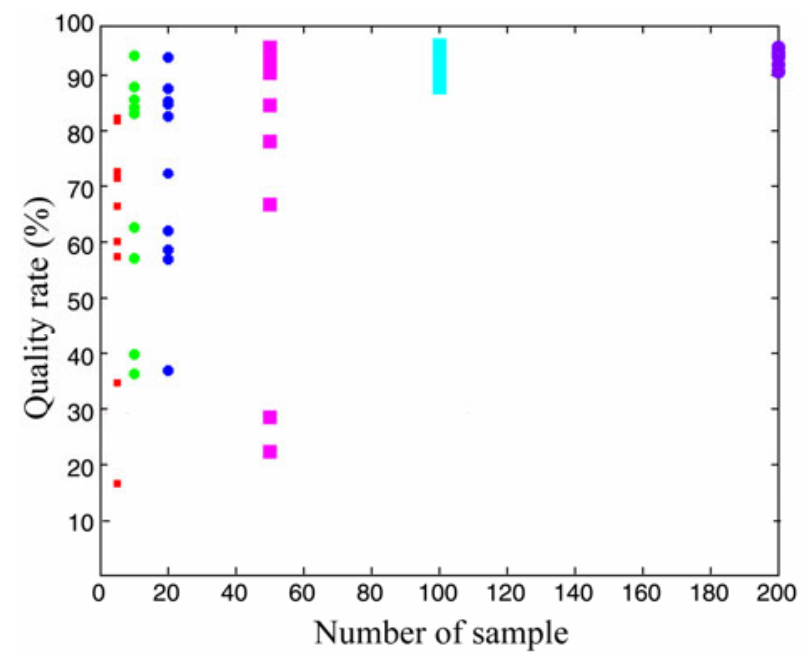

Fig. 8. Relationship between the number of training samples and the quality rate

\subsection{Size of PCBs}

Figure 9 shows the relationship between the size of PCB and the quality rate. The training samples were selected at random from the data set, and the same experiment was performed ten times in each size. The other experimental conditions were the same as Sec. 4. The result suggests that the quality rate is independent of the size of the PCB.

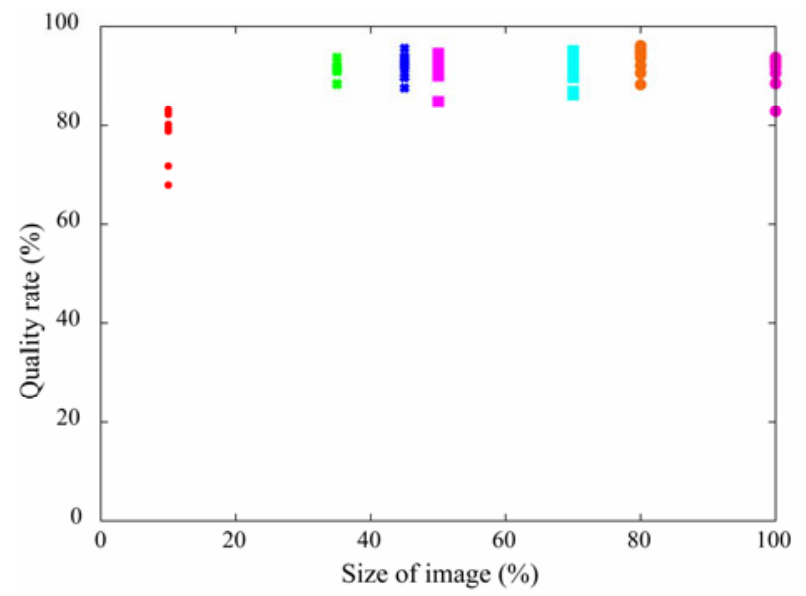

Fig. 9. Relationship between the PCB size and the quality rate 


\subsection{Standard Deviation of Gaussian Kernel}

In a Gaussian kernel function, the standard deviation that corresponds to the kernel width is a parameter related to the generalization ability. Figure 10 shows the relationship between the standard deviation and the quality rate. The number of training samples was 100 samples for each element. The standard deviation $\sigma$ at $\sigma \in[0,2]$ gives relatively stable quality rate to each element though metal line slightly changes.

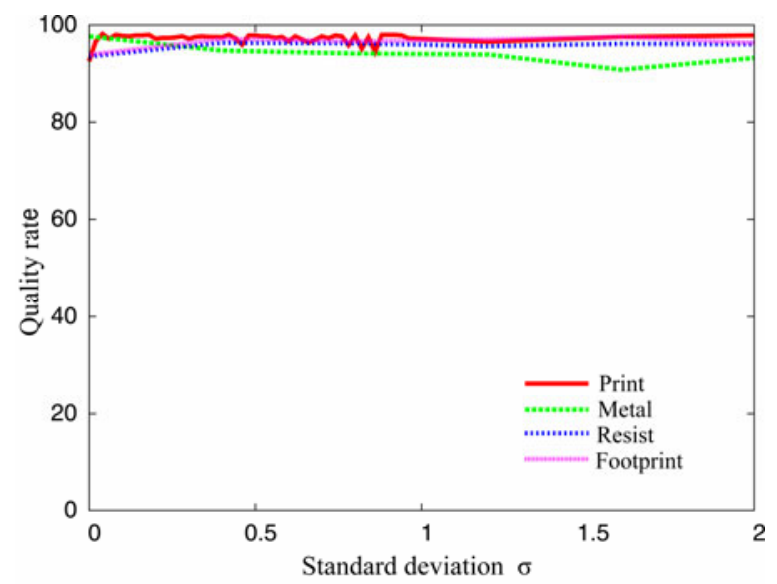

Fig. 10. Relationship between the standard deviation of the Gaussian kernel and the quality rate

\subsection{Feature Selection}

Table 2 shows change of the quality rate for the three channels selected at different wavelengths. Little change of the quality rate is recognized when selecting the channel in the visible range almost evenly. However, the result suggested that the best wavelength combination is $(450 \mathrm{~nm}, 550 \mathrm{~nm}, 650 \mathrm{~nm})$.

Table 2. Quality rate for the three channels selected at different wavelengths

\begin{tabular}{|c|c|c|c|}
\hline$\lambda_{1}(\mathrm{~nm})$ & $\lambda_{2}(\mathrm{~nm})$ & $\lambda_{3}(\mathrm{~nm})$ & Quality rate \\
\hline 430 & 530 & 630 & $92.4 \%$ \\
\hline 430 & 570 & 670 & $95.5 \%$ \\
\hline 440 & 530 & 630 & $93.3 \%$ \\
\hline 450 & 570 & 670 & $93.2 \%$ \\
\hline 450 & 550 & 650 & $96.4 \%$ \\
\hline 460 & 530 & 630 & $96.4 \%$ \\
\hline 470 & 530 & 630 & $95.3 \%$ \\
\hline
\end{tabular}




\section{Conclusion}

This paper has proposed an approach to a reliable material classification for PCBs by the kernel Fisher discriminant analysis. In this approach, reflectance values at only three different wavelengths reduced from the high-dimensional spectral imaging data were used for effectively classifying the surface material on each pixel point into several elements. We developed a kernel technique which constructs a subspace to maximize the class separability in a high-dimensional feature space. The performance of the proposed method was compared with the previous algorithms using the highdimensional spectral data. The goodness was shown in experiments, where the classification accuracy was compared with the unsupervised classification algorithms with high-dimensional features. Our algorithm could be applied directly to the material classification problem in a variety of raw PCBs.

Since the proposed algorithm uses only three-spectral channels, a color camera with narrow bands would be used as an imaging device. Selection of the best wavelength bands in the visible range remains as a future problem.

\section{References}

1. Moganti, F., Ercal, F., Dagli, C.H., Tsunekawa, S.: Automatic PCB Inspection Algorithms: A Survey. Computer Vision and Image Understanding 63(2), 287-313 (1996)

2. Chang, P.C., Chen, L.Y., Fan, C.Y.: A Case-based Evolutionary Model for Defect Classification of Printed Circuit Board Images. J. Intell. Manuf. 19, 203-214 (2008)

3. Tsai, D.M., Yang, R.H.: An Eigenvalue-based Similarity Measure and Its Application in Defect Detection. Image and Vision Computing 23(12), 1094-1101 (2005)

4. Ibrahim, Z., Al-Attas, S.A.R.: Wavelet-based Printed Circuit Board Inspection Algorithm. Integrated Computer-Aided Engineering 12, 201-213 (2005)

5. Huang, S.Y., Mao, C.W., Cheng, K.S.: Contour-Based Window Extraction Algorithm for Bare Printed Circuit Board Inspection. IEICE Trans. 88-D, 2802-2810 (2005)

6. Leta, F.R., Feliciano, F.F., Martins, F.P.R.: Computer Vision System for Printed Circuit Board Inspection. In: ABCM Symp. Series in Mechatronics, vol. 3, pp. 623-632 (2008)

7. Tominaga, S.: Material Identification via Multi-Spectral Imaging and Its Application to Circuit Boards. In: 10th Color Imaging Conference, Color Science, Systems and Applications, Scottsdale, Arizona, pp. 217-222 (2002)

8. Tominaga, S., Okamoto, S.: Reflectance-Based Material Classification for Printed Circuit Boards. In: 12th Int. Conf. on Image Analysis and Processing, Italy, pp. 238-243 (2003)

9. Ibrahim, A., Tominaga, S., Horiuchi, T.: Material Classification for Printed Circuit Boards by Spectral Imaging System. In: Trémeau, A., Schettini, R., Tominaga, S. (eds.) CCIW 2009. LNCS, vol. 5646, pp. 216-225. Springer, Heidelberg (2009)

10. Ibrahim, A., Tominaga, S., Horiuchi, T.: A Spectral Imaging Method for Material Classification and Inspection of Printed Circuit Boards. Optical Engineering 49(5), 057201-1-057201-10 (2010) 